\title{
Fractional Order Stochastic Differential Equation with Application in European Option Pricing
}

\author{
Qing Li, ${ }^{1}$ Yanli Zhou, ${ }^{2,3}$ Xinquan Zhao, ${ }^{1}$ and Xiangyu Ge ${ }^{1}$ \\ ${ }^{1}$ School of Statistics and Mathematics, Zhongnan University of Economics and Law, Wuhan 430073, China \\ ${ }^{2}$ School of Finance, Zhongnan University of Economics and Law, Wuhan 430073, China \\ ${ }^{3}$ Department of Mathematics and Statistics, Curtin University, Perth, WA 6845, Australia
}

Correspondence should be addressed to Yanli Zhou; ylzhou8507@gmail.com and Xiangyu Ge; xiangyu_ge@163.com

Received 20 January 2014; Revised 3 July 2014; Accepted 17 July 2014; Published 13 August 2014

Academic Editor: Jianping Li

Copyright (C) 2014 Qing Li et al. This is an open access article distributed under the Creative Commons Attribution License, which permits unrestricted use, distribution, and reproduction in any medium, provided the original work is properly cited.

\begin{abstract}
Memory effect is an important phenomenon in financial systems, and a number of research works have been carried out to study the long memory in the financial markets. In recent years, fractional order ordinary differential equation is used as an effective instrument for describing the memory effect in complex systems. In this paper, we establish a fractional order stochastic differential equation (FSDE) model to describe the effect of trend memory in financial pricing. We, then, derive a European option pricing formula based on the FSDE model and prove the existence of the trend memory (i.e., the mean value function) in the option pricing formula when the Hurst index is between 0.5 and 1. In addition, we make a comparison analysis between our proposed model, the classic Black-Scholes model, and the stochastic model with fractional Brownian motion. Numerical results suggest that our model leads to more accurate and lower standard deviation in the empirical study.
\end{abstract}

\section{Introduction}

Time series incorporating memory structure has been widely used in biological, chemical, and physical system. Memory effects also exist in financial systems. For example, the decision will be effected spontaneously by the past experience of decision makers. Plenty of financial variables with long memory effects have been found [1-4], such as the gross domestic product (GDP), interest rate, foreign exchange rates, stock price, and futures price. Garzareli et al. have proved the existence of memory effects in the stock price series by the conditional probability approach and measured the extent of long memory (autocorrelation) [5].

Memory effect is often measured by the autocorrelation function, and, recently, the Hurst index as an effective tool was introduced to measure the memory effect [6]. The Hurst index is often denoted by $H(0<H<1)$. In the case of $0<H<0.5$, time series has negative correlation and antipersistent behavior, which is called short-dependence memory. When $H=0.5$, the time series has no dependence. However, in the case of $0.5<H<1$, time series has positive correlation and persistent behavior, which is long-dependence memory. The persistent behavior was also called "Joseph Effect" by Mandelbrot and Wallis [7]. Cajueiro and Tabak $[8,9]$ have also found that memory effect exists in financial markets.

A number of researchers used fractional Brownian motion to depict the characteristic of memory. Mandelbrot and Van Ness first found that long memory effects exist in stock returns and gave the definition of fractional Brownian motion [10]. Since then, describing the memory by the fractional Brownian motion in financial market becomes more and more popular. For instance, Bęben and Orłowski [11], Huang and Yang [12], Evertsz [13], Lo [14], and Wen et al. $[15,16]$ have shown that the returns are of long-term (or shortterm) dependence in the markets. After Black and Scholes [17] developed the option pricing theory based on the classical stochastic differential equation, a large number of literatures studied the option price based on the fractional Brownian motion. For example, Necula [18], Rostek [19], and $\mathrm{Hu}$ and Øksendal [20] obtained the Black-Scholes option pricing formula under fractional Brownian motion. Ren et al. [21] have considered the option pricing model for $0.5<H<1$. 
In the case of $0<H<0.5$, the option pricing formula was studied by Wang et al. [22]. Chen et al. [23] established the mixed fractional-fractional version of Black-Scholes model with $0<H<1$ and gave the Ito's formula correspondingly.

However, the memory effects contain not only the noise memory effect but also the trend memory effect. Stochastic differential equation with fractional Brownian motion only describes the noise memory but cannot be used to study the trend memory effect of stock price. So we will describe the trend memory process by using the fractional derivative, which is another effective instrument to describe the memory effect. In particular, fractional calculus has been successfully applied in biology, physics, chemistry, and hydrology. Recently, the concept of fractal has been extended in financial mathematics [24]. This is due to the fact that fractional integral and derivatives can depict the memory and inherent process [25]. It has been realized that fractional derivative provides an excellent mathematical instrument for the description of complex process, irregular increment, memory properties, and intermediate process [25-28].

The fractional derivative is given as below:

$$
d^{\alpha} x=\mu(x, t) d t^{\alpha}
$$

where $\alpha$ is a fraction. This fractional differential equation is an appropriate mathematical approach to depict memory process of the increment. However, the fractional order derivative above only denotes the memory effect of a fixed process. Since the process in financial market has stochastic effect, we add stochastic process into fractional order ordinary differential equation. In this work, we propose a new model constructed by stochastic differential equation with fractional order. We denote the stochastic process of the asset price by fractional order stochastic differential equation as follows:

$$
d^{\alpha} x=\mu(x, t) d t^{\alpha}+\sigma(x, t) d B(t), \quad \alpha=2 H .
$$

In (2), $H$ is the Hurst index, which is an exponent describing the memory of the time series, and can be calculated by the $R / \mathrm{SD}$ analysis approach [6]. In the special case of $\alpha=$ 1 (i.e., $H=0.5$ ), the equation is reduced to the classic stochastic differential equation. Jumarie gave the Taylor's series of fractional order, expressed $d x$ in terms of fractional differential $d^{\alpha} x$ by using Taylor's series of fractional order, and, hence, obtained the expression of $x(t)$, which involves the so-called Mittag-Leffler function [29, 30]. Momani and Odibat presented the numerical approach of differential equation of fractional order [31]. Odibat proposed algorithms to compute the functions of fractional derivative [32].

The rest of this paper is organized as follows. Section 2 gives some basic concepts and theories on the fractional order ordinary differential equations and Hurst index and then establishes the fractional order stochastic differential equation in the financial market. In Section 3, based on the proposed stochastic differential equation with fractional order derivative, we give the corresponding Ito formula under the FSDE and then derive the fractional European option pricing formula. In Section 4, we conduct the empirical analysis of fractional order formula of stock price process by using the Monte Carlo simulation method, and we also make comparison analysis of option pricing formula under FSDE with the classic option pricing formula and option pricing formula based on fractional Brownian motion. The conclusions drawn from this study are presented in Section 5.

\section{Fractional Order Stochastic Differential Equation}

In this section, we first give some preliminaries about the fractional order ordinary differential equation and then expand them to the field of the stochastic differential equations. Thus, based on these previous research results, we can construct the generalized fractional order stochastic differential equation.

\subsection{Fractional Order Ordinary Differential Equations (FODE).} Now we introduce the definitions of fractional order integration and fractional order derivative. There exist several definitions of fractional derivatives, which are related to different applications. In our paper, we consider these two definitions, which are Riemann-Liouville integral and Caputo derivative [30].

Definition 1. $f(x)$ is a continuous function. Its RiemannLiouville fractional integral of order $\alpha$ of function $f(x)$ is defined as follows:

$$
I^{\alpha} f(x)=\frac{1}{\Gamma(\alpha)} \int_{0}^{x}(x-t)^{\alpha-1} f(t) d t \quad \alpha>0, x>0
$$

where $\alpha$ is a fraction and $\Gamma(\alpha)$ is the Gamma function with $\Gamma(\alpha)=\int_{0}^{\infty} x^{\alpha-1} \exp (-x) d x$.

Definition 2. Consider the function of Definition 1, and Caputo fractional derivative of order $\alpha$ of function $f(x)$ is defined as

$$
\begin{aligned}
\frac{d^{\alpha} f}{d x^{\alpha}} & =D^{\alpha} f(x)=I^{m-\alpha} D^{m} f(x) \\
& =\frac{1}{\Gamma(m-\alpha)} \int_{0}^{x}(x-t)^{m-\alpha-1} f^{(m)}(t) d t
\end{aligned}
$$

where $\alpha$ is a fraction, $m$ is an integer and $m=[\alpha]$ is the value of $\alpha$ rounded up to the nearest integer, and $f^{(m)}$ is the ordinary derivative of $f$.

Based on the definitions above, the following equality holds $[33,34]$ :

$$
f^{(\alpha)}(x)=\lim _{h \rightarrow 0} \frac{\Delta^{\alpha} f(x)}{h^{\alpha}} .
$$

In order to get the relations between the fractional derivative and ordinary derivative, we introduce the Taylor expansion of fractional order. 
Proposition 3. Assume that the continuous function $f(x)$ has fractional derivative of fractional order $k \alpha$, for any positive integer $k$ at any $\alpha, 0<\alpha<1$; then the following equality holds:

$$
f(x+h)=\sum_{k=0}^{\infty} \frac{h^{k \alpha} f^{(k \alpha)}(x)}{\Gamma(1+k \alpha)}, \quad 0<\alpha \leq 1,
$$

where $f^{(k \alpha)}$ is the derivative of order $\alpha k$ of $f(x)$, which can be denoted by $D^{k \alpha} f$.

Lemma 4. Assume that $m<\alpha<m+1, m \in N$; then,

$$
\begin{array}{r}
f^{(m)}(x+h)=\sum_{k=0}^{\infty} \frac{h^{k(\alpha-m)} D^{k(\alpha-m)} f^{(m)}(x)}{\Gamma[1+k(\alpha-m)]}, \\
m<\alpha<m+1 .
\end{array}
$$

Let $m$ be equal to 1 in (7), and take integration with respect to $h$; we then have the following result:

$$
\begin{aligned}
f(x+h)= & f(x)+h f^{\prime}(x) \\
& +\sum_{k=1}^{\infty} \frac{h^{1+k(\alpha-1)}}{\Gamma[2+k(\alpha-1)]} f^{(1+k(\alpha-1))}(x) .
\end{aligned}
$$

The proof of the lemma above can be found in [29].

By employing the fractional order Taylor formula and (5), we get the applications below. Given that $m$ is an integer with $m \geq 1$, the following results hold:

$$
\begin{array}{r}
f^{(\alpha)}(x)=\lim _{h \rightarrow 0} \frac{\Delta^{\alpha} f(x)}{h^{\alpha}}=\Gamma(1+\alpha) \lim _{h \rightarrow 0} \frac{\Delta f(x)}{h^{\alpha}}, \\
0<\alpha \leq 1, \\
f^{(\alpha)}(x)=\Gamma(1+(\alpha-m)] \lim _{h \rightarrow 0} \frac{\Delta f^{(m)}(x)}{h^{\alpha-m}}, \\
m<\alpha<m+1 \quad(1 \leq m) .
\end{array}
$$

We then compare the two equations, (8) and (9), when $1<$ $\alpha<2$; thus, the relationship between fractional difference and finite difference is obtained as follows:

(1) Discrete form:

$$
\Delta^{\alpha} f=\Gamma(1+\alpha) \Delta f, \quad 0<\alpha \leq 1
$$

Continuous form:

$$
d^{\alpha} f=\Gamma(1+\alpha) d f, \quad 0<\alpha \leq 1
$$

(2) Discrete form:

$$
\Delta^{\alpha} f=\Gamma(1+\alpha)\left[\Delta f-f^{\prime}(x) \Delta x\right], \quad 1<\alpha<2
$$

Continuous form:

$$
d^{\alpha} f=\Gamma(1+\alpha)\left[d f-f^{\prime}(x) d x\right], \quad 1<\alpha<2 .
$$

For the purpose of constructing the fractional order stochastic differential equations in this section, now we give some results of the integral with respect to $d t^{\alpha}$ in Lemma 5 presented below. Its detailed proof can be obtained in $[29,30]$.
Lemma 5. Let $f(t)$ denote a continuous function; then its integral with respect to $d t^{\alpha}$ is defined by the following equalities:

$$
\begin{gathered}
\int_{0}^{t} f(\tau)(d \tau)^{\alpha}=\alpha \int_{0}^{t}(t-\tau)^{\alpha-1} f(\tau) d \tau \quad 0<\alpha<1, \\
\text { (2) } \int_{0}^{t} f(\tau)(d \tau)^{\alpha}=\alpha(\alpha-1) \int_{0}^{t}(t-\tau)^{\alpha-2} F(\tau) d \tau \\
1<\alpha<2,
\end{gathered}
$$

where $F(t)=\int_{0}^{t} f(\tau) d \tau$; on making $f(t)=1$, we can have the result: $\int_{0}^{t} f(\tau)(d \tau)^{\alpha}=t^{\alpha}$.

2.2. Memory Effect and the Hurst Index. Time series $X_{t}=$ $\left\{X_{1}, X_{2}, \ldots, X_{N}\right\}$ is a stochastic process with $X_{t}$ recorded at the discrete times $t=0,1,2, \ldots, N$. A time series has the memory structure, if the lag period information affects the future changes. Time series displays long memory when the correlation between current and lag observations does not weaken to zero quickly over time.

Let $X_{t}$ be a stationary stochastic process with autocorrelation function $\rho(\tau), \tau=1,2, \ldots, m$, where $\tau$ denotes the time lag. If $\sum_{\tau=1}^{m}|\rho(\tau)|=\infty, X_{t}$ is called a long memory process; if $\sum_{\tau=1}^{m}|\rho(\tau)|<\infty, X_{t}$ is called a short memory process, and, otherwise, if $\rho(\tau)=0$, for $\tau \neq 0, X_{t}$ has no memory effect. The classical approach to measure the stochastic memory process is the autocorrelation function. Now, the Hurst index is widely used as an effective substitute of the autocorrelation function to determine long-range or short-range dependence.

The memory effect can be described by the memory parameter, namely, the Hurst index. Hurst index measures the smoothness of time series based on the asymptotic behavior of the rescale of the stochastic process. A key property of memory process is self-similarity, which is denoted by the Hurst index.

Definition 6. Stochastic process $X=\left\{X_{t}, t=1,2, \ldots, N\right\}$ is self-similar with Hurst index $H$ for any $a>0$ and at any time $t$; then we denote it by $X_{a t} \stackrel{d}{=} a^{H} X_{t}$, where Hurst index describes the self-similarity of stochastic process, and $\stackrel{d}{=}$ represents equality of the distribution.

In the following lemma, some basic properties are given and the corresponding proofs can be obtained in $[6,8]$.

Lemma 7. Suppose a time series $\left\{X_{t}, t=1,2, \ldots, N\right\}$ is selfsimilar with strictly stationary increment; then this time series has the following properties.

(1) The expectation of $X_{t}$ is $E\left[X_{t}\right]=0$ and, thus, $E\left[X_{t}^{2}\right]=$ $\sigma^{2}$ for all $t=1,2, \ldots, N$.

(2) The covariance function $\gamma(s, t)=E\left\{\left[X_{s}-E\left(X_{s}\right)\right]\left[X_{t}-\right.\right.$ $\left.\left.E\left(X_{t}\right)\right]\right\}=E\left[X_{s} X_{t}\right]$, which has the following result:

$$
\gamma(s, t)=\frac{\sigma^{2}}{2}\left[|s|^{2 H}-|s-t|^{2 H}+|t|^{2 H}\right] .
$$


(3) The autocovariance function of $X_{t}$ is given by $\gamma(\tau), \tau=$ $1,2, \ldots n$, where $\tau$ is the lag period:

$\gamma(\tau)=E\left[X_{t} X_{t+\tau}\right]=\frac{\sigma^{2}}{2}\left[|\tau+1|^{2 H}-2|\tau|^{2 H}+|\tau-1|^{2 H}\right]$.

(4) If $\tau \neq 0$, then we get the relationship between autocovariance function and Hurst index:

$$
\begin{array}{cc}
\gamma(\tau)=0, & H=0.5, \\
\gamma(\tau)<0, & 0<H<0.5, \\
\gamma(\tau)>0, & 0.5<H<1
\end{array}
$$

which means $\gamma(\tau)>0$ in the case of $0.5<H<1$; similarly, in the case $0<H<0.5, \gamma(\tau)<0$, and in the case $H=0.5$, $\gamma(\tau)=0$. According to the autocovariance function, we have that, in the case of $0<H<0.5$, the times series exhibit shortrange dependence; in the case of $H=0.5$, the times series has no dependence, which is a perfect random walk; and in the case of $0.5<H<1$, time series has long-range dependence.

The Hurst indexis usually estimated by the $R / S$ statistic approach. Given a stochastic process $\left\{X_{t}, t=1,2, \ldots, N\right\}$ of length $N$, we divide the time interval $N$ into $M$ contiguous subintervals of length $n$ such that $M \times n=N$. For each subinterval, the average value is $X_{n}=E\left[X_{t}\right]=(1 / n) \sum_{t=1}^{n} X_{t}$.

The running sum of the accumulated deviations from the mean is given as

$$
X_{t, n}=\sum_{t=1}^{k}\left(X_{t}-E\left[X_{t}\right]\right), \quad k=1,2, \ldots, n .
$$

The range over the time period $n$ is

$$
R(n)=\max X_{k, n}-\min X_{k, n}, \quad k=1,2, \ldots, n .
$$

The standard deviation of $X_{t}, t=1,2, \ldots, n$ is

$$
\operatorname{SD}(n)=\sqrt{\frac{1}{n-1} \sum_{t=1}^{n}\left(X_{t}-E\left[X_{t}\right]\right)^{2}}
$$

The rescaled range is $(R / \mathrm{SD})(n)=R(n) / \mathrm{SD}(n)$, and the relationship between $R / \mathrm{SD}$ statistic and $n$ is

$$
\left(\frac{R}{\mathrm{SD}}\right)(n)=\frac{R(n)}{\mathrm{SD}(n)}=\frac{1}{n} \sum_{i=1}^{n} \frac{R(i)}{\mathrm{SD}(i)}
$$

Thus, we can get the result:

$$
E\left[\frac{R(n)}{\mathrm{SD}(n)}\right]=\alpha n^{H}, \quad n \longrightarrow \infty
$$

where $\alpha$ is a constant and $H$ is the Hurst index.

As a consequence, we can get the Hurst index of the observed time by linear regression:

$$
\log E\left(\frac{R}{\mathrm{SD}}\right)(n)=H \log n+\log \alpha
$$

2.3. Fractional Order Stochastic Differential Equation (FSDE). Here, we generalize the classic stochastic differential equation to establish the fractional order stochastic differential equation based on the results presented before and then apply it to the option pricing in the next section.

Definition 8. Assuming that a financial asset price is $S$, according to the fractional ordinary differential equation, and considering the stochastic process, we can get the FSDE as follows:

$$
d^{\alpha} S=\mu(S, t)(d t)^{\alpha}+\sigma(S, t) d B(t), \quad \alpha=2 H,
$$

where $\mu(S, t)$ is the drift parameter, $\sigma(S, t)$ is the diffusion parameter, $d B(t)$ is the Wiener process, $d B(t)=\varepsilon \sqrt{d t}$, $\varepsilon \sim N(0,1)$ (normal distribution), and $d t$ and $d B(t)$ are uncorrelated, $d t d t=0, d t d B(t)=0, d B(t) d B(t)=d t$.

In a special case, suppose $\mu(S, t)=\mu S, \sigma(S, t)=\sigma S$, and then we have the linear stochastic differential equation:

$$
d^{\alpha} S=\mu S(d t)^{\alpha}+\sigma S d B(t), \quad \alpha=2 H .
$$

By using the results of (11), we can rewrite (22) into the following form of $d S$ with respect to $d t^{\alpha}$ :

$$
\begin{array}{r}
d S=\frac{\mu(S, t)}{\Gamma(1+\alpha)}(d t)^{\alpha}+\frac{\sigma(S, t)}{\Gamma(1+\alpha)} d B(t), \\
0<\alpha \leq 1, \quad 0<H \leq 0.5, \\
d S=\frac{\mu(S, t)}{\Gamma(1+\alpha)}(d t)^{\alpha}+\frac{\sigma(S, t)}{\Gamma(1+\alpha)} d B(t)+S^{\prime}(t) d t, \\
1<\alpha<2, \quad 0.5<H<1,
\end{array}
$$

where $S^{\prime}(t)$ is the first order derivative of $S$ about time $t$.

\section{European Call Option Pricing Based on FOSDE}

In this section, the corresponding Ito's formula and European call option pricing formula are derived based on the fractional order stochastic differential equation.

\subsection{Ito's Lemma Based on FSDE}

Lemma 9. Assume that the stock price S follows the fractional order stochastic differential equation as below:

$$
d^{\alpha} S=\mu S(d t)^{\alpha}+\sigma S d B(t), \quad \alpha=2 H
$$

then, the function $f=f\left(S_{t}, t\right)$ is still an Ito stochastic process, and the following expressions hold.

When $0.25<H \leq 0.5$

$$
\begin{aligned}
d f= & {\left[\frac{\partial f}{\partial t}+\frac{\sigma^{2} S^{2}}{2 \Gamma^{2}(1+\alpha)} \frac{\partial^{2} f}{\partial S^{2}}\right] d t+\frac{\mu S}{\Gamma(1+\alpha)} \frac{\partial f}{\partial S}(d t)^{2 H} } \\
& +\frac{\sigma S}{\Gamma(1+\alpha)} \frac{\partial f}{\partial S} d B(t) .
\end{aligned}
$$


When $0.5<H<1$

$$
\begin{aligned}
d f= & {\left[\frac{\partial f}{\partial t}+\frac{\sigma^{2} S^{2}}{2 \Gamma^{2}(1+\alpha)} \frac{\partial^{2} f}{\partial S^{2}}+S^{\prime}(t) \frac{\partial f}{\partial S}\right] d t } \\
& +\frac{\mu S}{\Gamma(1+\alpha)} \frac{\partial f}{\partial S}(d t)^{2 H}+\frac{\sigma S}{\Gamma(1+\alpha)} \frac{\partial f}{\partial S} d B(t) .
\end{aligned}
$$

Proof. According to the Ito formula, we notice that

$$
\begin{aligned}
\Delta f= & \frac{\partial f}{\partial t} \Delta t+\frac{\partial f}{\partial S} \Delta S+\frac{1}{2} \frac{\partial^{2} f}{\partial S^{2}}(\Delta S)^{2}+\frac{\partial^{2} f}{\partial S \partial t}(\Delta S \Delta t) \\
& +\frac{1}{2} \frac{\partial^{2} f}{\partial t^{2}}(\Delta t)^{2}
\end{aligned}
$$

and the discrete form of $d^{\alpha} S=\mu S(d t)^{\alpha}+\sigma S d B(t)$ is $\Delta^{\alpha} S=$ $\mu S(\Delta t)^{\alpha}+\sigma S \Delta B(t)$.

In this paper, we only consider the case that $0.25<H<1$. There are two reasons for this consideration: first, the Hurst index $H$ is much larger than 0 generally; second, when $0.25<$ $H<1, \alpha=2 H>0.5,(\Delta t)^{2 \alpha}$ and $(\Delta t)^{\alpha+0.5}$ are infinitesimal. Hence, we do not need to consider the case of $0<H \leq 0.25$.

(1) In the case of $0.25<H \leq 0.5$, since $E(\varepsilon)=0, E\left(\varepsilon^{2}\right)=$ 1 , we have

$$
\begin{gathered}
\Delta S=\frac{\mu S}{\Gamma(1+\alpha)}(\Delta t)^{\alpha}+\frac{\sigma S}{\Gamma(1+\alpha)} \varepsilon(\Delta t)^{1 / 2}, \\
\Delta S \Delta t=\frac{\mu S}{\Gamma(1+\alpha)}(\Delta t)^{\alpha+1}+\frac{\sigma S}{\Gamma(1+\alpha)} \varepsilon(\Delta t)^{1.5} \longrightarrow 0, \\
(\Delta S)^{2}=\frac{\mu^{2} S^{2}}{\Gamma^{2}(1+\alpha)}(\Delta t)^{2 \alpha}+\frac{\sigma^{2} S^{2}}{\Gamma^{2}(1+\alpha)} \varepsilon^{2}(\Delta t) \\
+\frac{2 \mu \sigma S^{2}}{\Gamma^{2}(1+\alpha)} \varepsilon(\Delta t)^{\alpha+0.5} \longrightarrow \frac{\sigma^{2} S^{2}}{\Gamma^{2}(1+\alpha)}(\Delta t) .
\end{gathered}
$$

According to the Ito formula presented above, we can get

$$
\begin{aligned}
\Delta f= & \frac{\partial f}{\partial t} \Delta t+\frac{\partial f}{\partial S} \Delta S+\frac{1}{2} \frac{\partial^{2} f}{\partial S^{2}}(\Delta S)^{2}+\frac{\partial^{2} f}{\partial S \partial t}(\Delta S \Delta t) \\
& +\frac{1}{2} \frac{\partial^{2} f}{\partial t^{2}}(\Delta t)^{2} \\
= & \frac{\partial f}{\partial t} \Delta t+\frac{\partial f}{\partial S} \Delta S+\frac{1}{2} \frac{\partial^{2} f}{\partial S^{2}}(\Delta S)^{2} \\
= & \frac{\partial f}{\partial t} \Delta t+\frac{\partial f}{\partial S}\left[\frac{\mu S}{\Gamma(1+\alpha)}(\Delta t)^{\alpha}+\frac{\sigma S}{\Gamma(1+\alpha)} \varepsilon(\Delta t)^{0.5}\right] \\
& +\frac{\sigma^{2} S^{2}}{2 \Gamma^{2}(1+\alpha)} \frac{\partial^{2} f}{\partial S^{2}}(\Delta t) \\
= & {\left[\frac{\partial f}{\partial t}+\frac{\sigma^{2} S^{2}}{2 \Gamma^{2}(1+\alpha)} \frac{\partial^{2} f}{\partial S^{2}}\right] \Delta t+\frac{\mu S}{\Gamma(1+\alpha)} \frac{\partial f}{\partial S}(\Delta t)^{2 H} } \\
& +\frac{\sigma S}{\Gamma(1+\alpha)} \frac{\partial f}{\partial S} \varepsilon(\Delta t)^{0.5} .
\end{aligned}
$$

Thus, the differential form is given below:

$$
\begin{aligned}
d f= & {\left[\frac{\partial f}{\partial t}+\frac{\sigma^{2} S^{2}}{2 \Gamma^{2}(1+\alpha)} \frac{\partial^{2} f}{\partial S^{2}}\right] d t+\frac{\mu S}{\Gamma(1+\alpha)} \frac{\partial f}{\partial S}(d t)^{2 H} } \\
& +\frac{\sigma S}{\Gamma(1+\alpha)} d B(t) .
\end{aligned}
$$

(2) In the case of $0.5<H<1$

$$
\begin{aligned}
& \Delta S=\frac{\mu S}{\Gamma(1+\alpha)}(\Delta t)^{\alpha}+\frac{\sigma S}{\Gamma(1+\alpha)} \varepsilon(\Delta t)^{0.5}+S^{\prime}(t) \Delta t, \\
& \Delta S \Delta t=\frac{\mu S}{\Gamma(1+\alpha)}(\Delta t)^{\alpha+1}+\frac{\sigma S}{\Gamma(1+\alpha)} \varepsilon(\Delta t)^{1.5} \\
& +S^{\prime}(t)(\Delta t)^{2} \longrightarrow 0, \\
& (\Delta S)^{2}=\frac{\mu^{2} S^{2}}{\Gamma^{2}(1+\alpha)}(\Delta t)^{2 \alpha}+\frac{\sigma^{2} S^{2}}{\Gamma^{2}(1+\alpha)} \varepsilon^{2} \Delta t+\left[S^{\prime}(t)\right]^{2}(\Delta t)^{2} \\
& +\frac{2 \mu \sigma S^{2}}{\Gamma^{2}(1+\alpha)} \varepsilon(\Delta t)^{\alpha+0.5}+\frac{2 \mu S S^{\prime}(t)}{\Gamma(1+\alpha)}(\Delta t)^{\alpha+1} \\
& +\frac{2 \sigma S S^{\prime}(t)}{\Gamma(1+\alpha)} \varepsilon(\Delta t)^{1.5} \longrightarrow \frac{\sigma^{2} S^{2}}{\Gamma^{2}(1+\alpha)} \Delta t, \\
& \Delta f=\frac{\partial f}{\partial t} \Delta t+\frac{\partial f}{\partial S} \Delta S+\frac{1}{2} \frac{\partial^{2} f}{\partial S^{2}}(\Delta S)^{2} \\
& =\frac{\partial f}{\partial t} \Delta t+\frac{\partial f}{\partial S}\left[\frac{\mu S}{\Gamma(1+\alpha)}(\Delta t)^{\alpha}+\frac{\sigma S}{\Gamma(1+\alpha)} \varepsilon(\Delta t)^{0.5}\right. \\
& \left.+S^{\prime}(t) \Delta t\right]+\frac{\sigma^{2} S^{2}}{2 \Gamma^{2}(1+\alpha)} \frac{\partial^{2} f}{\partial S^{2}} \Delta t \\
& =\left[\frac{\partial f}{\partial t}+\frac{\sigma^{2} S^{2}}{2 \Gamma^{2}(1+\alpha)} \frac{\partial^{2} f}{\partial S^{2}}+S^{\prime}(t) \frac{\partial f}{\partial S}\right] \Delta t \\
& +\frac{\mu S}{\Gamma(1+\alpha)} \frac{\partial f}{\partial S}(\Delta t)^{2 H}+\frac{\sigma S}{\Gamma(1+\alpha)} \frac{\partial f}{\partial S} \varepsilon(\Delta t)^{0.5} \text {. }
\end{aligned}
$$

Similarly, we obtain the differential form as follows:

$$
\begin{aligned}
d f= & {\left[\frac{\partial f}{\partial t}+\frac{\sigma^{2} S^{2}}{2 \Gamma^{2}(1+\alpha)} \frac{\partial^{2} f}{\partial S^{2}}+S^{\prime}(t) \frac{\partial f}{\partial S}\right] d t } \\
& +\frac{\mu S}{\Gamma(1+\alpha)} \frac{\partial f}{\partial S}(d t)^{2 H}+\frac{\sigma S}{\Gamma(1+\alpha)} \frac{\partial f}{\partial S} d B(t)
\end{aligned}
$$

To price a European option, we first introduce Lemma 10, which connects the fractional order stochastic differential equations to the partial differential equations. 
Lemma 10. $f(S(t), t)$ is the solution of the partial differential equations:

$$
\begin{gathered}
\frac{\partial f}{\partial t}+r S \frac{\partial f}{\partial S}+\frac{\sigma^{2} S^{2}}{2 \Gamma^{2}(1+\alpha)} \frac{\partial^{2} f}{\partial S^{2}}-r f=0, \quad 0.25<H \leq 0.5, \\
f(S(T), T)=f(S(T)), \\
\frac{\partial f}{\partial t}+\left[S^{\prime}(t)+r S\right] \frac{\partial f}{\partial S}+\frac{\sigma^{2} S^{2}}{2 \Gamma^{2}(1+\alpha)} \frac{\partial^{2} f}{\partial S^{2}}-r f=0, \\
0.5<H<1, \\
f(S(T), T)=f(S(T)) .
\end{gathered}
$$

Proof. First, make portfolios $\Pi=\Delta S-f$ and $d \Pi=\Delta d S-d f$.

(1) In the case of $0.25<H \leq 0.5$,

$$
\begin{aligned}
d \Pi= & \Delta d S-d f \\
= & \Delta\left[\frac{\mu S}{\Gamma(1+\alpha)} d t^{\alpha}+\frac{\sigma S}{\Gamma(1+\alpha)} d B(t)\right] \\
& -\left[\left[\frac{\partial f}{\partial t}+\frac{\sigma^{2} S^{2}}{2 \Gamma^{2}(1+\alpha)} \frac{\partial^{2} f}{\partial S}\right] d t\right. \\
& \left.+\left[\frac{\mu S}{\Gamma(1+\alpha)} \frac{\partial f}{\partial S}\right](d t)^{2 H}+\frac{\sigma S}{\Gamma(1+\alpha)} \frac{\partial f}{\partial S} d B(t)\right] \\
= & -\left[\frac{\partial f}{\partial t}+\frac{\sigma^{2} S^{2}}{2 \Gamma^{2}(1+\alpha)} \frac{\partial^{2} f}{\partial S}\right] d t \\
& +\frac{\mu S}{\Gamma(1+\alpha)}\left(\Delta-\frac{\partial f}{\partial S}\right)(d t)^{2 H} \\
& +\frac{\sigma S}{\Gamma(1+\alpha)}\left(\Delta-\frac{\partial f}{\partial S}\right) d B(t) .
\end{aligned}
$$

When $\Delta=\partial f / \partial S$, we can get the riskless asset portfolio

$$
d \Pi=\Delta d S-d f=-\left[\frac{\partial f}{\partial t}+\frac{\sigma^{2} S^{2}}{2 \Gamma^{2}(1+\alpha)} \frac{\partial^{2} f}{\partial S^{2}}\right] d t .
$$

And because the portfolio $\Pi$ is riskless, according to the Bellman Equation, we have $d \Pi=r \Pi d t$, where $r$ is the riskless rate. Thus, we get the equation $d \Pi=r \Pi d t=-[(\partial f / \partial t)+$ $\left.\left(\sigma^{2} S^{2} / 2 \Gamma^{2}(1+\alpha)\right)\left(\partial^{2} f / \partial S^{2}\right)\right] d t$. Consequently, we obtain the first partial differential equation

$$
\frac{\partial f}{\partial t}+r S \frac{\partial f}{\partial S}+\frac{\sigma^{2} S^{2}}{2 \Gamma^{2}(1+\alpha)} \frac{\partial^{2} f}{\partial S^{2}}-r f=0 .
$$

(2) In the case of $0.5<H<1$,

$$
\begin{aligned}
\Delta \Pi= & \Delta d S-d f \\
= & \Delta\left[\frac{\mu S}{\Gamma(1+\alpha)}(d t)^{\alpha}+\frac{\sigma S}{\Gamma(1+\alpha)} d B(t)+S^{\prime}(t) d t\right] \\
- & \left\{\left[\frac{\partial f}{\partial t}+\frac{\sigma^{2}}{2 \Gamma^{2}(1+\alpha)} \frac{\partial^{2} f}{\partial S^{2}}+x^{\prime}(t) \frac{\partial f}{\partial S}\right] d t\right. \\
& \left.+\frac{\mu}{\Gamma(1+\alpha)} \frac{\partial f}{\partial S}(d t)^{2 H}+\frac{\sigma}{\Gamma(1+\alpha)} \frac{\partial f}{\partial S} d B(t)\right\} \\
=- & {\left[\frac{\partial f}{\partial t}+\frac{\sigma^{2}}{2 \Gamma^{2}(1+\alpha)} \frac{\partial^{2} f}{\partial S^{2}}+S^{\prime}(t) \frac{\partial f}{\partial S}\right] d t } \\
+ & \frac{\mu S}{\Gamma(1+\alpha)}\left(\Delta-\frac{\partial f}{\partial S}\right)(d t)^{2 H} \\
+ & \frac{\sigma S}{\Gamma(1+\alpha)}\left(\Delta-\frac{\partial f}{\partial S}\right) d B(t) .
\end{aligned}
$$

When $\Delta=\partial f / \partial S$, we can also get the riskless asset portfolio

$$
d \Pi=\Delta d S-d f=-\left[\frac{\partial f}{\partial t}+\frac{\sigma^{2}}{2 \Gamma^{2}(1+\alpha)} \frac{\partial^{2} f}{\partial S^{2}}+S^{\prime}(t) \frac{\partial f}{\partial S}\right] d t .
$$

And again because $\Pi$ is riskless, we can get the equation

$$
d \Pi=r \Pi d t=-\left[\frac{\partial f}{\partial t}+\frac{\sigma^{2}}{2 \Gamma^{2}(1+\alpha)} \frac{\partial^{2} f}{\partial S^{2}}+S^{\prime}(t) \frac{\partial f}{\partial S}\right] d t .
$$

Similarly, the second partial differential equation can be obtained as below:

$$
\frac{\partial f}{\partial t}+\left[S^{\prime}(t)+r S\right] \frac{\partial f}{\partial S}+\frac{\sigma^{2} S^{2}}{2 \Gamma^{2}(1+\alpha)} \frac{\partial^{2} f}{\partial S^{2}}-r f=0 .
$$

3.2. European Call Option Based on FSDE. Before we proceed to price the European call option, we make the assumptions as below:

(1) $r$ is the riskless rate and is a constant;

(2) the exchange of the stock is continuous and the stock can be divided;

(3) the tax of the stock exchange is free;

(4) the bonus of the stock cannot be paid within the duration of derivatives;

(5) no arbitrage exists in the market;

(6) the price of stock follows a fractional order stochastic differential equation

$$
\frac{d^{\alpha} S}{S}=r(d t)^{\alpha}+\sigma d B(t), \quad \alpha=2 H ;
$$


(7) the strike price is $K$;

(8) the maturity is $T$,

where $S$ is the price of the stock and $r$ is the riskless interest rate; $\sigma$ is the volatility of the price of stock; $H$ is Hurst parameter of the stock.

In the following work, we will derive the fractional option pricing formula based on the risk-neutral assumption. If the price of underlying asset is subject to the geometric Brownian motion and the return $\mu$ is equal to the riskless interest rate $r$ (i.e., $\mu=r$ ), we have

$$
\frac{d^{\alpha} S}{S}=r(d t)^{\alpha}+\sigma d B(t), \quad \alpha=2 H
$$

(1) In the case of $0.25<H \leq 0.5$, according to Ito's Lemma 9, we can get the price of the stock as

$$
\begin{aligned}
d(\ln S)= & \frac{r}{\Gamma(1+\alpha)}(d t)^{2 H}-\frac{\sigma^{2}}{2 \Gamma^{2}(1+\alpha)} d t \\
& +\frac{\sigma}{\Gamma(1+\alpha)} d B(t), \quad 0.25<H \leq 0.5 .
\end{aligned}
$$

Integrate (44) and use Lemma 5; then, we can get the solution of $S$ :

$$
\begin{gathered}
S_{T}=S \exp \left(\frac{r}{\Gamma(1+\alpha)}\left(T^{2 H}-t^{2 H}\right)-\frac{\sigma^{2}}{2 \Gamma^{2}(1+\alpha)}(T-t)\right. \\
\left.+\frac{\sigma}{\Gamma(1+\alpha)}(B(T)-B(t))\right) .
\end{gathered}
$$

Therefore, the European call option pricing formula follows:

$$
c=S e^{(r / \Gamma(1+\alpha))\left(T^{2 H}-t^{2 H}\right)-r(T-t)} N\left(d_{1}\right)-K e^{-r(T-t)} N\left(d_{2}\right),
$$

where

$$
\begin{array}{r}
d_{1}=\left(\Gamma(1+\alpha) \ln \frac{S}{K}+r\left(T^{2 H}-t^{2 H}\right)\right. \\
\left.\quad+\frac{\sigma^{2}}{2 \Gamma(1+\alpha)}(T-t)\right)(\sigma \sqrt{T-t})^{-1} \\
d_{2}=\left(\Gamma(1+\alpha) \ln \frac{S}{K}+r\left(T^{2 H}-t^{2 H}\right)\right. \\
\left.\quad-\frac{\sigma^{2}}{2 \Gamma(1+\alpha)}(T-t)\right)(\sigma \sqrt{T-t})^{-1} .
\end{array}
$$

Proof. The price of the European option is given by $c=$ $e^{-r(T-t)} E\left[\max \left(S_{T}-K\right), 0\right]$, where $E(\cdot)$ is the expectation of the option price based on risk-neutral, and the price of the asset $S_{T}$ obeys the lognormal distribution:

$$
\begin{aligned}
\ln S_{T}- & \ln S \\
\sim N( & \frac{r}{\Gamma(1+\alpha)}\left(T^{2 H}-t^{2 H}\right) \\
& \left.\quad-\frac{\sigma^{2}}{2 \Gamma^{2}(1+\alpha)}(T-t), \frac{\sigma^{2}}{\Gamma^{2}(1+\alpha)}(T-t)\right) .
\end{aligned}
$$

Let $W=\left(\ln S_{T}-m\right) / s$; obviously, $W \sim N(0,1)$, and the probability density function $h(W)=(1 / \sqrt{2 \pi}) e^{-W^{2} / 2}$, where $m=E\left(\ln S_{T}\right)=\ln S+(r / \Gamma(1+\alpha))\left(T^{2 H}-t^{2 H}\right)-\left(\sigma^{2} / 2 \Gamma^{2}(1+\right.$ $\alpha)(T-t)$, and $s=\sigma \sqrt{T-t} / \Gamma(1+\alpha)$. Hence,

$$
\begin{aligned}
E[\max & \left.\left(S_{T}-K, 0\right)\right] \\
= & \int_{-\infty}^{+\infty} \max \left(S_{T}-K, 0\right) h\left(S_{T}\right) d S(T) \\
= & \int_{K}^{+\infty}\left(S_{T}-K\right) h\left(S_{T}\right) d S_{T}+\int_{-\infty}^{K} 0 h\left(S_{T}\right) d S_{T} \\
= & \int_{\ln K}^{+\infty}\left(e^{S_{T}}-K\right) h\left(\ln S_{T}\right) d\left(\ln S_{T}\right) \\
= & \int_{(\ln K-m) / s}^{+\infty}\left(e^{S_{T}}-K\right) h\left(\frac{\ln S_{T}-m}{s}\right) d\left(\frac{\ln S_{T}-m}{s}\right) \\
= & \int_{(\ln K-m) / s}^{+\infty}\left(e^{S_{T}}-K\right) h\left(\ln S_{T}\right) d\left(\ln S_{T}\right) \\
= & \int_{(\ln K-m) / s}^{+\infty}\left(e^{s W+m}-K\right) h(W) d W \\
= & \int_{(\ln K-m) / s}^{+\infty} e^{\left(s^{2} / 2\right)+m} \frac{1}{\sqrt{2 \pi}} e^{-(W-s)^{2} / 2} d W \\
= & -K e^{(r / \Gamma(1+\alpha))\left(T^{2 H}-t^{2 H}\right)} N\left(d_{1}\right)-K N\left(d_{2}\right), \\
& \left.-\frac{m-\ln K}{s}\right)
\end{aligned}
$$

where

$$
\begin{array}{r}
d_{1}=\left(\Gamma(1+\alpha) \ln \frac{S}{K}+r\left(T^{2 H}-t^{2 H}\right)\right. \\
\left.\quad+\frac{\sigma^{2}}{2 \Gamma(1+\alpha)}(T-t)\right)(\sigma \sqrt{T-t})^{-1}, \\
d_{2}=\left(\Gamma(1+\alpha) \ln \frac{S}{K}+r\left(T^{2 H}-t^{2 H}\right)\right. \\
\left.\quad-\frac{\sigma^{2}}{2 \Gamma(1+\alpha)}(T-t)\right)(\sigma \sqrt{T-t})^{-1} .
\end{array}
$$


So we get the European option pricing formula as follows:

$$
\begin{aligned}
c= & e^{-r(T-t)} E\left[\max \left(S_{T}-K, 0\right)\right] \\
= & e^{-r(T-t)}\left[S e^{(r / \Gamma(1+\alpha))\left(T^{2 H}-t^{2 H}\right)} N\left(d_{1}\right)-K N\left(d_{2}\right)\right] \\
= & S e^{(r / \Gamma(1+\alpha))\left(T^{2 H}-t^{2 H}\right)-r(T-t)} N\left(d_{1}\right) \\
& -K e^{-r(T-t)} N\left(d_{2}\right) .
\end{aligned}
$$

(2) In the case of $0.5<H<1$, in a similar way, according to Ito's Lemma 9, the price of the stock is

$$
\begin{aligned}
d(\ln S)= & \frac{r}{\Gamma(1+\alpha)}(d t)^{2 H}+\left(\frac{S^{\prime}}{S}-\frac{\sigma^{2}}{2 \Gamma^{2}(1+\alpha)}\right) d t \\
& +\frac{\sigma}{\Gamma(1+\alpha)} d B(t) .
\end{aligned}
$$

Notice that $\left(S^{\prime} / S\right) d t=d(\ln S)=\ln S_{t+1}-\ln S_{t}=m(t), m(t)$ represents the daily logarithm returns of stock $S$, and $m(t)=$ $\mu(t) d t, \mu(t)$ is the returns of one year; thus, $S^{\prime} / S=\mu(t)$, and (52) can be written as below:

$$
\begin{aligned}
d(\ln S)= & \frac{r}{\Gamma(1+\alpha)}(d t)^{2 H}+\left(\mu(t)-\frac{\sigma^{2}}{2 \Gamma^{2}(1+\alpha)}\right) d t \\
& +\frac{\sigma}{\Gamma(1+\alpha)} d B(t) .
\end{aligned}
$$

By integrating (52) and employing Lemma 5, we get the solution of $S$ :

$$
\begin{aligned}
S_{T}=S \exp [ & \frac{r}{\Gamma(1+\alpha)}\left(T^{2 H}-t^{2 H}\right)+\int_{t}^{T} \mu(s) d s \\
& \left.-\frac{\sigma^{2}}{2 \Gamma^{2}(1+\alpha)}(T-t)+\frac{\sigma}{\Gamma(1+\alpha)}(B(T)-B(t))\right] .
\end{aligned}
$$

Consequently, the European call option pricing formula is obtained:

$$
\begin{aligned}
c= & S e^{(r / \Gamma(1+\alpha))\left(T^{2 H}-t^{2 H}\right)+\int_{t}^{T} \mu(s) d s-r(T-t)} N\left(d_{1}\right) \\
& -K e^{-r(T-t)} N\left(d_{2}\right),
\end{aligned}
$$

where

$$
\begin{aligned}
d_{1}= & \left(\Gamma(1+\alpha) \ln \frac{S}{K}+\int_{t}^{T} \mu(s) d s\right. \\
& \left.+r\left(T^{2 H}-t^{2 H}\right)+\frac{\sigma^{2}}{2 \Gamma(1+\alpha)}(T-t)\right)(\sigma \sqrt{T-t})^{-1}, \\
d_{2}= & \left(\Gamma(1+\alpha) \ln \frac{S}{K}+\int_{t}^{T} \mu(s) d s+r\left(T^{2 H}-t^{2 H}\right)\right. \\
& \left.\quad-\frac{\sigma^{2}}{2 \Gamma(1+\alpha)}(T-t)\right)(\sigma \sqrt{T-t})^{-1} .
\end{aligned}
$$

Proof. Let $W=\left(\ln S_{T}-m\right) / s$, and it is obvious that $W \sim$ $N(0,1)$, so the probability density function $h(W)=(1 /$ $\sqrt{2 \pi}) e^{-W^{2} / 2}$, where

$$
\begin{aligned}
m= & \widehat{E}\left(\ln S_{T}\right) \\
= & \ln S+\int_{t}^{T} \mu(s) d s-\frac{\sigma^{2}}{2 \Gamma^{2}(1+\alpha)}(T-t) \\
& +\frac{r}{\Gamma(1+\alpha)}\left(T^{2 H}-t^{2 H}\right), \quad s=\frac{\sigma(T-t)^{0.5}}{\Gamma(1+\alpha)} .
\end{aligned}
$$

Hence,

$$
\begin{aligned}
E[\max & \left.\left(S_{T}-K, 0\right)\right] \\
= & \int_{-\infty}^{+\infty} \max \left(S_{T}-K, 0\right) h\left(S_{T}\right) d S_{T} \\
= & \int_{K}^{+\infty}\left(S_{T}-K\right) h\left(S_{T}\right) d S_{T} \\
= & \int_{(\ln K-m) / s}^{+\infty}\left(e^{s W+m}-K\right) h(W) d W \\
= & \int_{(\ln K-m) / s}^{+\infty} e^{\left(s^{2} / 2\right)+m} \frac{1}{\sqrt{2 \pi}} e^{-(W-s)^{2} / 2} d W \\
& -K N\left(\frac{m-\ln K}{s}\right) \\
= & S e^{\int_{t}^{T} \mu(s) d s+(r / \Gamma(1+\alpha))\left(T^{2 H}-t^{2 H}\right)} N\left(d_{1}\right)-K N\left(d_{2}\right),
\end{aligned}
$$

where

$$
\begin{gathered}
d_{1}=\left(\Gamma(1+\alpha) \ln \frac{S}{K}+\int_{t}^{T} \mu(s) d s+r\left(T^{2 H}-t^{2 H}\right)\right. \\
\left.\quad+\frac{\sigma^{2}}{2 \Gamma(1+\alpha)}(T-t)\right)(\sigma \sqrt{T-t})^{-1}, \\
d_{2}=\left(\Gamma(1+\alpha) \ln \frac{S}{K}+\int_{t}^{T} \mu(s) d s+r\left(T^{2 H}-t^{2 H}\right)\right. \\
\left.-\frac{\sigma^{2}}{2 \Gamma(1+\alpha)}(T-t)\right)(\sigma \sqrt{T-t})^{-1} .
\end{gathered}
$$

Finally, the European option pricing formula is given as below:

$$
\begin{aligned}
c= & e^{-r(T-t)} E\left[\max \left(S_{T}-K, 0\right)\right] \\
= & e^{-r(T-t)}\left[S e^{\int_{t}^{T} \mu(s) d s+(r / \Gamma(1+\alpha))(T-t)^{2 H}} N\left(d_{1}\right)-K N\left(d_{2}\right)\right] \\
= & S e^{(r / \Gamma(1+\alpha))\left(T^{2 H}-t^{2 H}\right)+\int_{t}^{T} \mu(s) d s-r(T-t)} N\left(d_{1}\right) \\
& -K e^{-r(T-t)} N\left(d_{2}\right) .
\end{aligned}
$$


From the result we derived, the option price formula contains mean value function $\int_{t}^{T} \mu(s) d s$ of the logarithmic returns of stock price, which is the effect of trend memory. Therefore, we proved that trend memory exists in the financial systems.

Now, we give the European call option pricing formula under the risk-neutral measure. Let the mean returns of stock be equal to the riskless rate $r$; by taking the expectation of the returns in case $0.5<H<1$, we have $E[\mu(t)]=\mu=r$, where $r$ is the riskless returns. Then, we simplify the mean value function $\int_{t}^{T} \mu(s) d s=\int_{t}^{T} \mu d s=\mu(T-t)=r(T-t)$ and have $\mu-r=0$; thus, we get the option pricing formula

$$
c=S e^{(r / \Gamma(1+\alpha))\left(T^{2 H}-t^{2 H}\right)} N\left(d_{1}\right)-K e^{-r(T-t)} N\left(d_{2}\right),
$$

where

$$
\begin{aligned}
d_{41}=( & \Gamma(1+\alpha) \ln \frac{S}{K}+r\left(T^{2 H}-t^{2 H}\right) \\
& \left.+\left(r+\frac{\sigma^{2}}{2 \Gamma(1+\alpha)}\right)(T-t)\right)(\sigma \sqrt{T-t})^{-1}, \\
d_{42}= & \left(\Gamma(1+\alpha) \ln \frac{S}{K}+r\left(T^{2 H}-t^{2 H}\right)\right. \\
& \left.+\left(r-\frac{\sigma^{2}}{2 \Gamma(1+\alpha)}\right)(T-t)\right)(\sigma \sqrt{T-t})^{-1} .
\end{aligned}
$$

\section{Results and Discussion}

To explain the memory effects in financial market, we make some comparisons in this section between our proposed European option pricing model and its underlying stock price equation and the well-known classic models, such as the Black-Scholes model (Black and Scholes (1973) [17]) and Black-Scholes model under fractional Brownian motion (Necula (2002) [18], Hu and Øksendal (2003) [20]).

4.1. Comparing European Pricing Formula with Other Models.

(1) Classical Black-Scholes model: $d S=r S d t+\sigma S d B(t)$ [17].

The European option pricing formula is $c=S N\left(d_{11}\right)-$ $K e^{-r(T-t)} N\left(d_{12}\right)$, where

$$
\begin{aligned}
& d_{11}=\frac{\ln (S / K)+\left(r+\sigma^{2} / 2\right)(T-t)}{\sigma \sqrt{T-t}}, \\
& d_{12}=\frac{\ln (S / K)+\left(r-\sigma^{2} / 2\right)(T-t)}{\sigma \sqrt{T-t}} .
\end{aligned}
$$

The classical Black-Scholes model was established under the assumption that the price process is Markov process and that the price process is independent and has no memory effect; however, the memory effects exist in price process.

(2) SDE with fractional Brownian motion: $d S=r S d t+$ $\sigma S d B_{H}(t)[18,20]$.
The European option pricing formula is $c=S N\left(d_{21}\right)-$ $K e^{-r(T-t)} N\left(d_{22}\right)$, where

$$
\begin{aligned}
& d_{21}=\frac{\ln (S / K)+r(T-t)+\left(\sigma^{2} / 2\right)\left(T^{2 H}-t^{2 H}\right)}{\sigma\left(T^{H}-t^{H}\right)}, \\
& d_{22}=\frac{\ln (S / K)+r(T-t)-\left(\sigma^{2} / 2\right)\left(T^{2 H}-t^{2 H}\right)}{\sigma\left(T^{H}-t^{H}\right)} .
\end{aligned}
$$

The fractional Brownian motion model has improved the Black-Scholes model by considering the memory effect of the asset price but only considered the memory effect of the noise.

(3) Fractional order SDE (FSDE model): $d^{\alpha} S=r S(d t)^{\alpha}+$ $\sigma S d B(t), \alpha=2 H$.

In the case of $0.25<H \leq 0.5$, the European call option pricing formula is

$$
c=S e^{(r / \Gamma(1+\alpha))(T-t)^{2 H}-r(T-t)} N\left(d_{31}\right)-K e^{-r(T-t)} N\left(d_{32}\right),
$$

where

$$
\begin{aligned}
d_{31}= & \left(\Gamma(1+\alpha) \ln \frac{S}{K}+r\left(T^{2 H}-t^{2 H}\right)\right. \\
& \left.+\frac{\sigma^{2}}{2 \Gamma(1+\alpha)}(T-t)\right)(\sigma \sqrt{T-t})^{-1}, \\
d_{32}= & \left(\Gamma(1+\alpha) \ln \frac{S}{K}+r\left(T^{2 H}-t^{2 H}\right)\right. \\
& \left.-\frac{\sigma^{2}}{2 \Gamma(1+\alpha)}(T-t)\right)(\sigma \sqrt{T-t})^{-1} .
\end{aligned}
$$

When $H=0.5$, the option formula is reduced to the classic option formula.

In the case of $0.5<H<1$, the European call option pricing formula is

$$
c=S e^{(r / \Gamma(1+\alpha))\left(T^{2 H}-t^{2 H}\right)} N\left(d_{41}\right)-K e^{-r(T-t)} N\left(d_{42}\right),
$$

where

$$
\begin{aligned}
d_{41}=( & \Gamma(1+\alpha) \ln \frac{S}{K}+r\left(T^{2 H}-t^{2 H}\right) \\
& \left.+\left(r+\frac{\sigma^{2}}{2 \Gamma(1+\alpha)}\right)(T-t)\right)(\sigma \sqrt{T-t})^{-1}, \\
d_{42}=( & \Gamma(1+\alpha) \ln \frac{S}{K}+r\left(T^{2 H}-t^{2 H}\right) \\
& \left.+\left(r-\frac{\sigma^{2}}{2 \Gamma(1+\alpha)}\right)(T-t)\right)(\sigma \sqrt{T-t})^{-1} .
\end{aligned}
$$

In the paper, our model takes a new memory effect into consideration, which is called the trend memory effect of the asset price. 
TABLE 1: Simulation results of SDE model.

\begin{tabular}{lccccc}
\hline Simulation times & Real value & Mean value & Standard deviation & Error rate & Confidence interval \\
\hline 100 & 2445 & 2351.9 & 198.6958 & $3.81 \%$ & {$[2313,2390.8]$} \\
1000 & 2445 & 2358.7 & 195.7074 & $3.53 \%$ & {$[2346.5,2370.8]$} \\
10000 & 2445 & 2357 & 200.5667 & $3.60 \%$ & {$[2353.1,2360.9]$} \\
100000 & 2445 & 2360 & 202.2802 & $3.48 \%$ & {$[2358.7,2361.2]$} \\
\hline
\end{tabular}

TABLE 2: Simulation results of SDE with FBM.

\begin{tabular}{lccccc}
\hline Simulation times & Real value & Mean value & Standard deviation & Error rate & Confidence interval \\
\hline 100 & 2445 & 2358.2 & 77.4242 & $3.55 \%$ & {$[2343.0,2373.3]$} \\
1000 & 2445 & 2360.8 & 76.3254 & $3.44 \%$ & {$[2356.1,2365.6]$} \\
10000 & 2445 & 2360 & 78.0423 & $3.48 \%$ & {$[2358.6,2361.6]$} \\
100000 & 2445 & 2361.2 & 78.5882 & $3.43 \%$ & {$[2360.8,2361.7]$} \\
\hline
\end{tabular}

TABLE 3: Simulation results of FSDE.

\begin{tabular}{lccccc}
\hline Simulation times & Real value & Mean value & Standard deviation & Error rate & Confidence interval \\
\hline 100 & 2445 & 2362.7 & 19.0566 & $3.37 \%$ & {$[2359.1,2366.5]$} \\
1000 & 2445 & 2368.4 & 21.1279 & $3.13 \%$ & {$[2367.2,2369.8]$} \\
10000 & 2445 & 2367.0 & 19.7396 & $3.19 \%$ & {$[2366.7,2367.5]$} \\
100000 & 2445 & 2369.5 & 20.4312 & $3.09 \%$ & {$[2369.5,2369.7]$} \\
\hline
\end{tabular}

4.2. Comparing the Asset Price Equation with Other Models. The underlying asset price equations to be used to make comparison are given as follows:

(1) the SDE model: $d S=r S d t+\sigma S d B(t)$ [17].

The stock price equation is $S_{T}=S \exp \left[\left(r-\sigma^{2} / 2\right)(T-\right.$ $t)+\sigma \Delta B(t)]$

(2) the SDE model with fractional Brownian motion: $d S=r S d t+\sigma S d B_{H}(t)[18,20]$.

The stock price equation is $S_{T}=S \exp \left[r(T-t)-\sigma^{2}(T-\right.$ $\left.t)^{2 H}+\sigma \Delta B_{H}(t)\right]$

(3) the fractional order SDE model: $d^{\alpha} S=r S(d t)^{\alpha}+$ $\sigma S d B(t), \alpha=2 H$

when $0.25<H \leq 0.5$, the stock price equation is

$$
\begin{aligned}
S_{t}=S_{t-1} \exp [ & \frac{r}{\Gamma(1+\alpha)} \Delta t^{2 H}-\frac{\sigma^{2}}{2 \Gamma^{2}(1+\alpha)} \Delta t \\
& \left.+\frac{\sigma}{\Gamma(1+\alpha)} \varepsilon \Delta B(t)\right] .
\end{aligned}
$$

However, when $0.5<H<1$, the stock price equation is

$$
\begin{gathered}
S_{t}=S_{t-1} \exp \left[\frac{r}{\Gamma(1+\alpha)} \Delta t^{2 H}+\left(r-\frac{\sigma^{2}}{2 \Gamma^{2}(1+\alpha)}\right) \Delta t\right. \\
\left.+\frac{\sigma}{\Gamma(1+\alpha)} \varepsilon \Delta B(t)\right] .
\end{gathered}
$$

To illustrate the proposed FSDE model, we simulate the three types of stochastic differential equations presented above by using the Monte Carlo simulation method and then make comparison of these three different models. The data used in the empirical analysis is the daily closing price index series of CSI300 index of China. The time range is from January 4, 2012 , to October 27, 2012, with the initial value $S_{0}=2299$ (the price index on January 4, 2012) and the final value $S_{T}=2445$. We choose the one-year bonds interest rate $r=2.65 \%$ in China as the riskless rate and the mean yield $\mu=0.0266$. The Hurst parameter is $H=0.6614$, which is estimated by $R / S$ analysis approach. Given a 95 percent confidence interval, the simulation results are shown in Tables 1, 2, and 3.

From the results in Tables $1-3$, by using Monte Carlo simulation, we conclude that the error of our proposed FSDE model is smaller than the conventional SDE model and SDE with FBM model. If we take the simulating process 100000 times, which is large enough for the error analysis, we obtain that the error rate of SDE model is $3.48 \%$, the SDE driven by fractional Brownian motion model is $3.43 \%$, and the FSDE model is $3.09 \%$, respectively.

In addition, the standard derivation of simulation is also much lower than the SDE model and SDE with FBM model, and the confidence interval is smaller than those two classic models. In the same way, when we simulate 100000 times, the standard deviation of SDE model is 202.2802, the SDE driven by fractional Brownian motion model is 78.5882, and FSDE model is 20.4312. Thus, we get the conclusion that the FSDE has about 10 times lower standard derivation than the SDE model and about 2.5 times lower standard derivation than the SDE driven by fractional Brownian motion model.

\section{Conclusions and Future Research}

Because the fractional order ordinary differential equations can capture the memory effect in the financial system, 
we established the fractional order stochastic differential equation by adding the stochastic process into the fractional ordinary differential equation. Based on this stochastic differential equation with fractional order, we apply the fractional order stochastic differential equation to the financial market. We constructed the stock price $d^{\alpha} S=\mu(S, t) d t^{\alpha}+$ $\sigma(S, t) d B(t)$, where $\alpha=2 H, H$ is Hurst index, and derived the stock price process in the cases of $0.25<H \leq 0.5$ and $0.5<$ $H<1$, respectively, and the European call option pricing formula under the fractional order stochastic differential equation. From the European option pricing formula, we find the trend memory in stock price process when Hurst index is between 0.5 and 1 .

In addition, we made some comparisons in terms of the pricing option formula and its underlying stock price process between our proposed approach and the other two classical models. We find that the new approach leads to a better result than the classic approach and fractional Brownian motion approach when we simulate the stock prices by Monte Carlo simulation.

It would be an interesting work if we improve our model by connecting fractional ordinary differential equation with fractional Brownian motion, which can describe both the trend memory and the noise memory.

\section{Conflict of Interests}

The authors declare that there is no conflict of interests regarding the publication of this paper.

\section{Acknowledgments}

The first author acknowledges the scholarship funds for the funds for fostering one hundred best Ph.D. degree papers (2013YB1901) provided by Zhongnan University of Economics and Law and also acknowledges the support of the School of Mathematics Science in Monash University for International Joint Training for Ph.D. This research was supported by the Chinese National Social Science Foundation (10BJY104).

\section{References}

[1] E. Panas, "Long memory and chaotic models of prices on the London metal exchange," Resources Policy, vol. 27, no. 4, pp. 485-490, 2001.

[2] B. J. West and S. Picozzi, "Fractional Langevin model of memory in financial time series," Physical Review E-Statistical, Nonlinear, and Soft Matter Physics, vol. 65, no. 3, Article ID 037106, 2002.

[3] F. Wen and Z. Liu, "A copula-based correlation measure and its application in chinese stock market," International Journal of Information Technology and Decision Making, vol. 8, no. 4, pp. 787-801, 2009.

[4] F. Wen, X. Gong, Y. Chao, and X. Chen, "The effects of prior outcomes on risky choice: evidence from the stock market," Mathematical Problems in Engineering, vol. 2014, Article ID 272518, 8 pages, 2014.
[5] F. Garzareli, M. Cristelli, A. Zaccaria, and L. Pietronero, "Memory effects in stock price dynamics: evidence of technical trading," Scientific Reports, vol. 4, article 4487, 2014.

[6] H. E. Hurst, "Long-term storage capacity of reservoirs," Transactions of the American Society of Civil Engineers, vol. 116, pp. 770-799, 1951.

[7] B. B. Mandelbrot and J. R. Wallis, "Noah, Joseph and operational hydrology," Water Resources Research, vol. 4, no. 5, pp. 909-918, 1968.

[8] D. O. Cajueiro and B. M. Tabak, "The Hurst exponent over time: testing the assertion that emerging markets are becoming more efficient," Physica A: Statistical Mechanics and its Applications, vol. 336, no. 3-4, pp. 521-537, 2004.

[9] D. O. Cajueiro and B. M. Tabak, "Ranking efficiency for emerging markets," Chaos, Solitons and Fractals, vol. 22, no. 2, pp. 349352, 2004.

[10] B. B. Mandelbrot and J. W. Van Ness, "Fractional brownian motions, fractional noises and applications," Society for Industrial and Applied Mathematics, vol. 10, no. 4, pp. 422-437, 1968.

[11] M. Bęben and A. Orłowski, "Correlations in financial time series: established versus emerging markets," The European Physical Journal B, vol. 20, no. 4, pp. 527-530, 2001.

[12] B. Huang and C. W. Yang, "The fractional structure in multinational stock returns," Applied Economics Letters, vol. 2, pp. 67-71, 1995.

[13] C. J. G. Evertsz, "Fractional geometry of financial time series," Fractals, vol. 3, pp. 609-616, 1995.

[14] A. W. Lo, "Long term memory in stock market prices," Econometrica, vol. 59, pp. 1279-1313, 1991.

[15] F. Wen and X. Yang, "Skewness of return distribution and coefficient of risk premium," Journal of Systems Science and Complexity, vol. 22, no. 3, pp. 360-371, 2009.

[16] F. Wen, Z. He, and X. Chen, "Investors' risk preference characteristics and conditional skewness," Mathematical Problems in Engineering, vol. 2014, Article ID 814965, 14 pages, 2014.

[17] F. Black and M. Scholes, "The pricing of option and corporate liabilities," Journal of Political Economy, vol. 81, pp. 637-659, 1973.

[18] C. Necula, "Option pricing in a fractional Brownian motion environment," Social Science Research Network, 2002.

[19] S. Rostek, Option Pricing in Fractional Brownian Markets, vol. 622 of Lecture Notes in Economics and Mathematical Systems, Academic Press, Springer, Berlin, Germany, 2009.

[20] Y. Hu and B. Øksendal, "Fractional white noise calculus and applications to finance," Infinite Dimensional Analysis, Quantum Probability and Related Topics, vol. 6, no. 1, pp. 1-32, 2003.

[21] F. Ren, X. Wang, and J. Liang, "A proof for French's empirical formula on option pricing," Chaos, Solitons and Fractals, vol. 12, no. 13, pp. 2441-2453, 2001.

[22] X. Wang, W. Qiu, and F. Ren, "Option pricing of fractional version of the Black-Scholes model with Hurst exponent $\mathrm{H}$ being in (1/3, 1/2 )," Chaos, Solitons and Fractals, vol. 12, no. 3, pp. 599-608, 2001.

[23] J. Chen, F. Ren, and W. Qiu, "Option pricing of a mixed fractional-fractional version of the Black-Scholes model," Chaos, Solitons and Fractals, vol. 21, no. 5, pp. 1163-1174, 2004.

[24] W. Chen, "Nonlinear dynamics and chaos in a fractional-order financial system," Chaos, Solitons and Fractals, vol. 36, no. 5, pp. 1305-1314, 2008.

[25] I. Pollubny, Fractional Differential Equations, Academic Press, San Diego, Calif, USA, 1999. 
[26] S. Das, Functional Fractional Calculus for System Identification and Controls, Springer, Berlin, Germany, 2008.

[27] R. Caponetto, G. Dongola, L. Fortuna, and I. Petras, Fractional Order Systems: Modeling and Control Applications, World Scientific, River Edge, NJ, USA, 2010.

[28] C. A. Monje, Y. Chen, B. Vinagre, D. Xue, and V. Feliu, Fractional-Order Systems and Controls: Fundamentals and Applications, Springer, London, UK, 2010.

[29] G. Jumarie, "On the representation of fractional Brownian motion as an integral with respect to $(\mathrm{d} t)^{a}$," Applied Mathematics Letters, vol. 18, no. 7, pp. 739-748, 2005.

[30] G. Jumarie, "On the solution of the stochastic differential equation of exponential growth driven by fractional Brownian motion," Applied Mathematics Letters: An International Journal of Rapid Publication, vol. 18, no. 7, pp. 817-826, 2005.

[31] S. Momani and Z. Odibat, "Numerical comparison of methods for solving linear differential equations of fractional order," Chaos, Solitons and Fractals, vol. 31, no. 5, pp. 1248-1255, 2007.

[32] Z. M. Odibat, "Computational algorithms for computing the fractional derivatives of functions," Mathematics and Computers in Simulation, vol. 79, no. 7, pp. 2013-2020, 2009.

[33] G. Jumarie, "Stochastic differential equations with fractional Brownian motion input," International Journal of Systems Science: Principles and Applications of Systems and Integration, vol. 24, no. 6, pp. 1113-1131, 1993.

[34] G. Jumarie, "Fractional Brownian motions via random walk in the complex plane and via fractional derivative. Comparison and further results on their Fokker-Planck equations," Chaos, Solitons and Fractals, vol. 22, no. 4, pp. 907-925, 2004. 


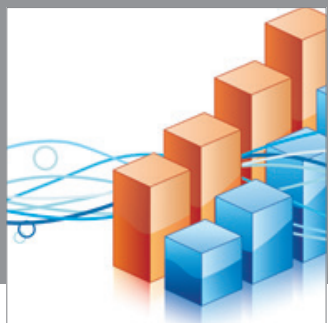

Advances in

Operations Research

mansans

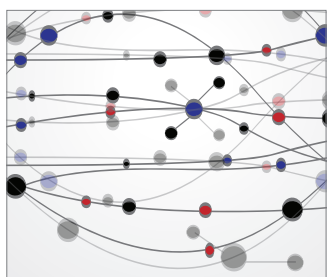

The Scientific World Journal
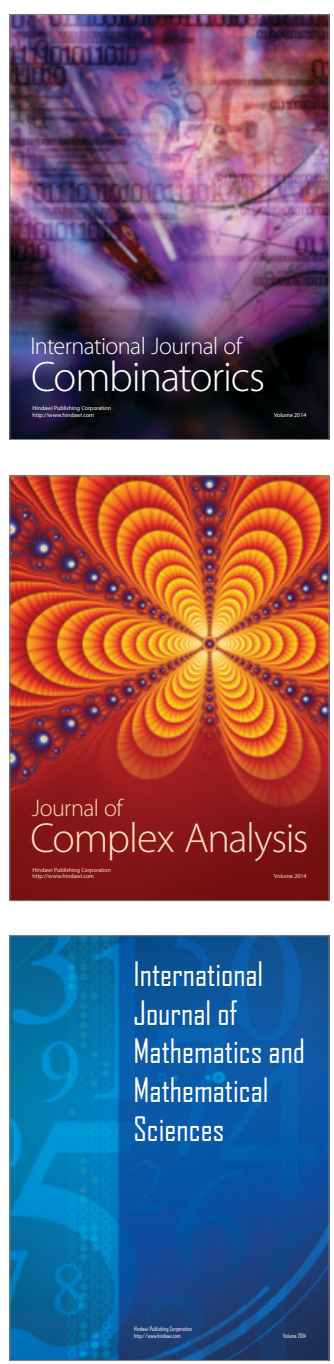
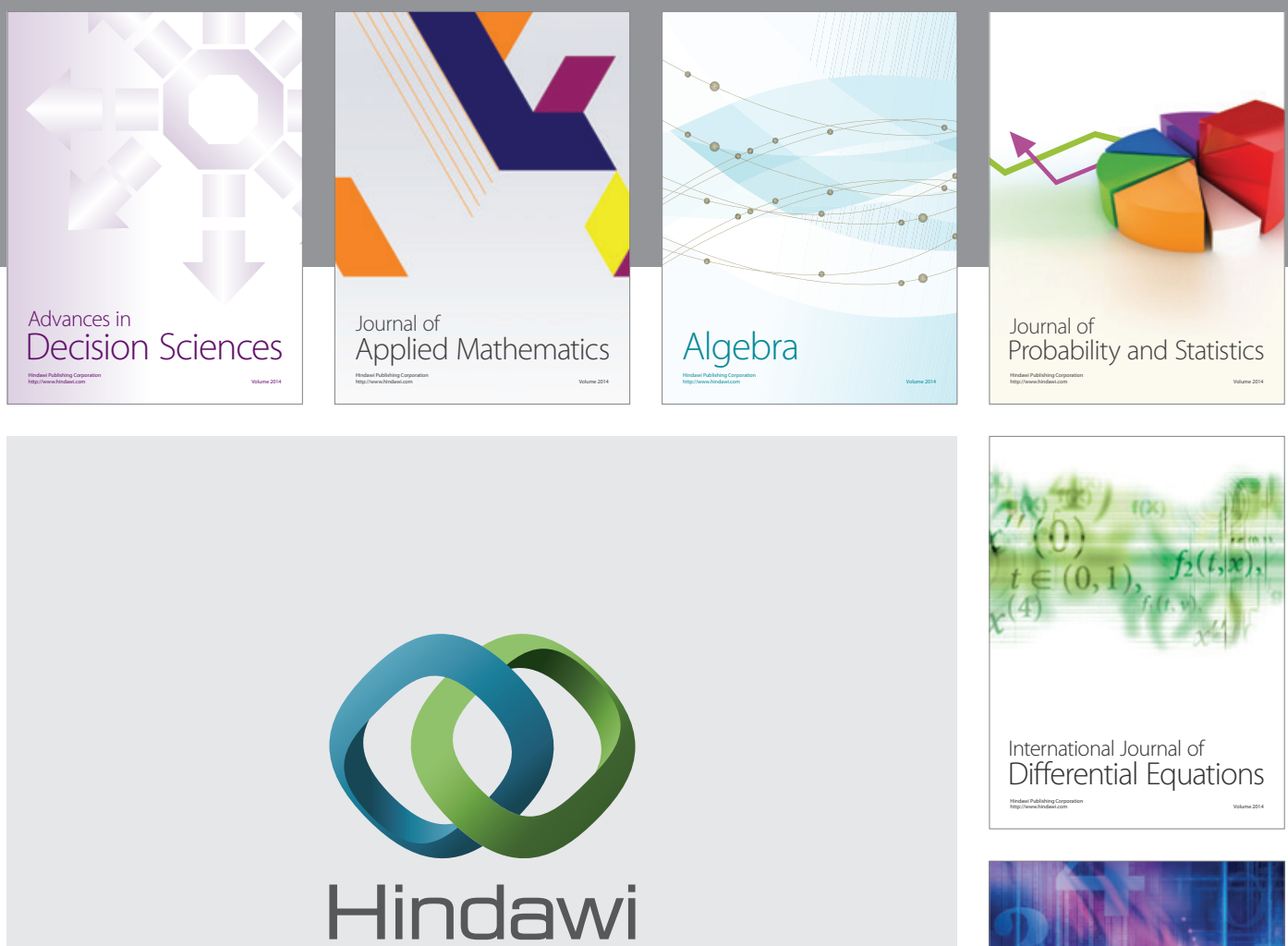

Submit your manuscripts at http://www.hindawi.com
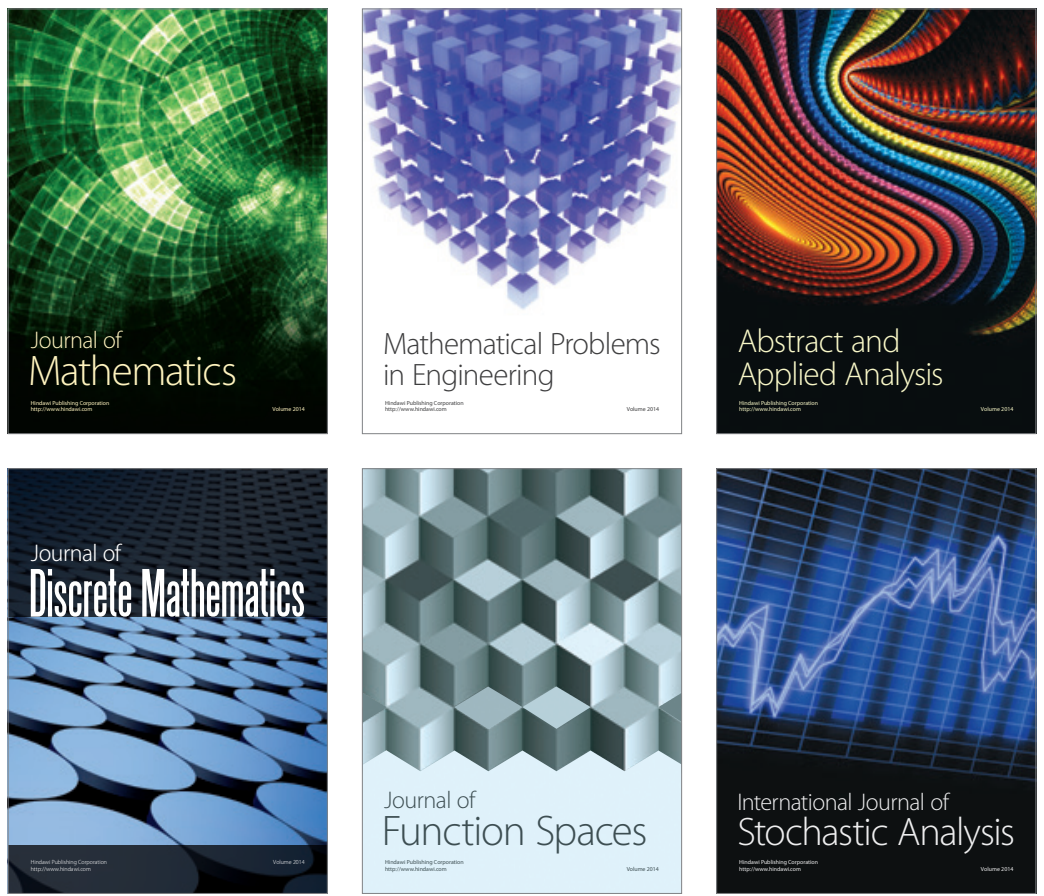

Journal of

Function Spaces

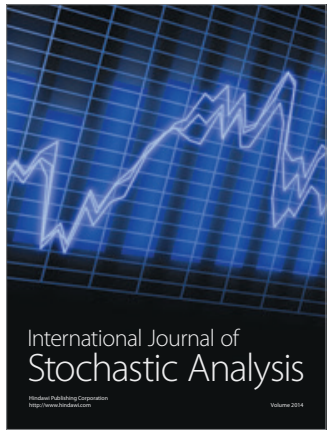

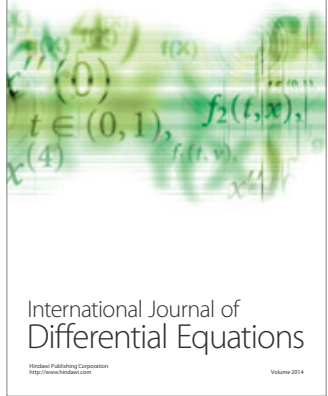
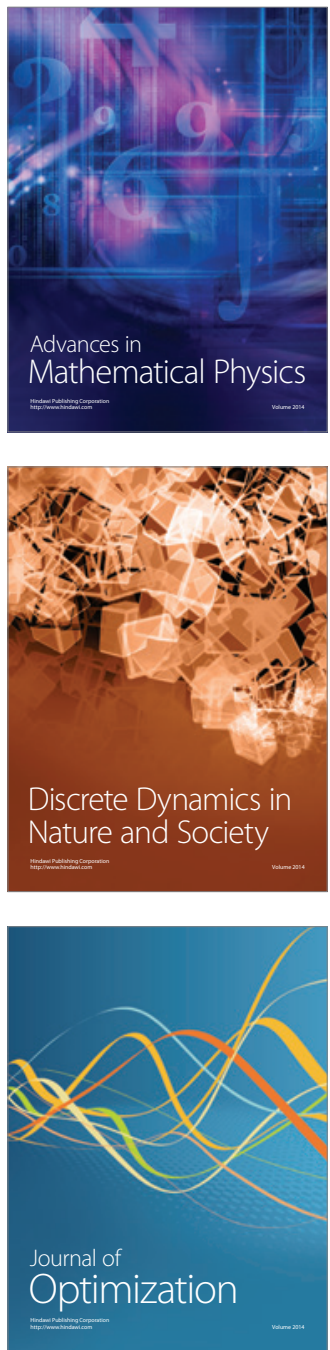